\title{
Transparent and robust amphiphobic surfaces exploiting nanohierarchical surface-grown metal-organic frameworks
}

Vikramjeet Singh ${ }^{1}$, Xuehu Men ${ }^{1}$, Manish K. Tiwari ${ }^{1,2 *}$

${ }^{1}$ Nanoengineered Systems Laboratory, UCL Mechanical Engineering, University College London, London, WC1E 7JE, UK.

${ }^{2}$ Wellcome/EPSRC Centre for Interventional and Surgical Sciences, University College London, London, W1W 7TS, UK

*Corresponding author. Email: m.tiwari@ucl.ac.uk

\section{Contents}

${ }^{*}$ Corresponding author. Email: m.tiwari@ucl.ac.uk................................................................ 1

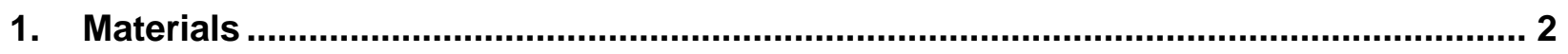

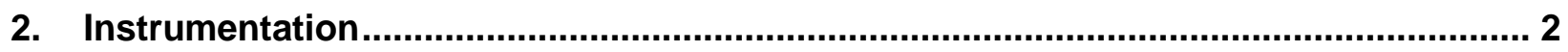

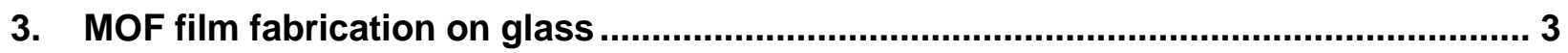

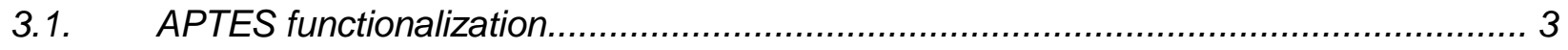

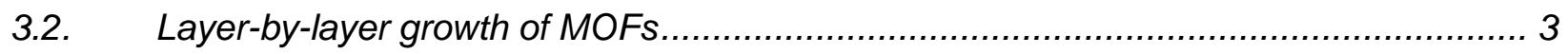

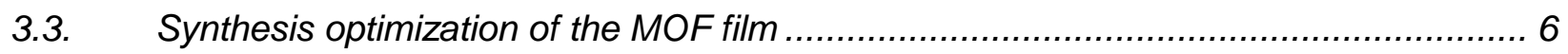

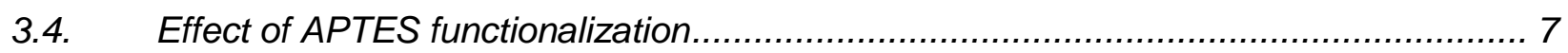

3.5. Post-synthesis functionalization of MOFs........................................................... 7

4. Fabrication of comparative surfaces................................................................... 8

4.1. Liquid infused MOFs for comparison .............................................................. 8

4.2. Fabrication of slippery omniphobic (SOCAL) coating …...................................... 8

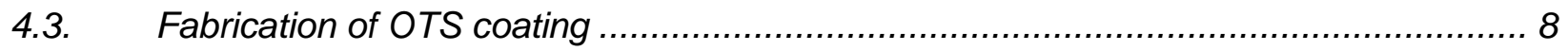

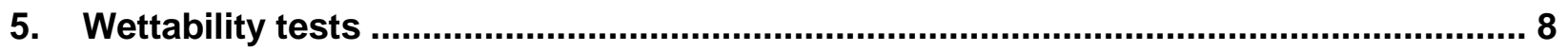

6. Solid fraction of the MOF surface

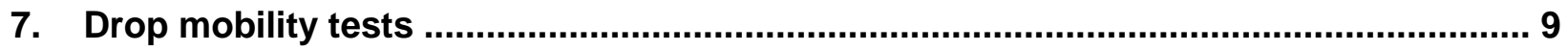

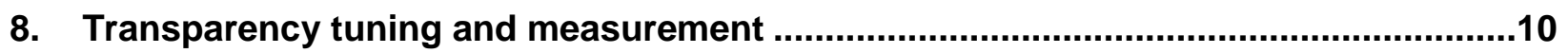

9. Theoretical droplet sliding velocity calculations .........................................................11

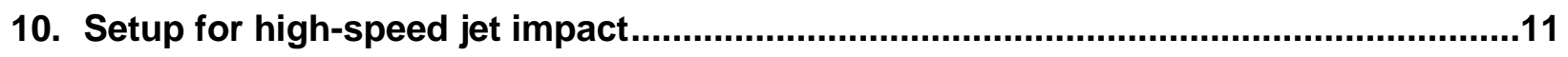

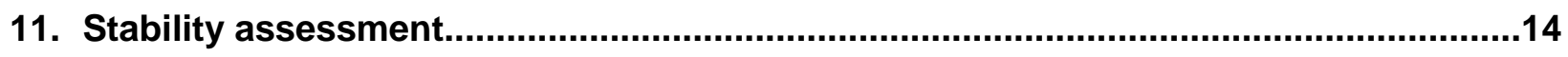

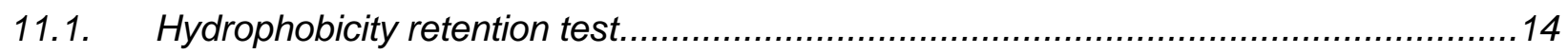

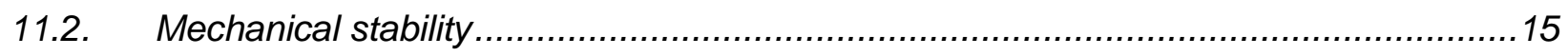




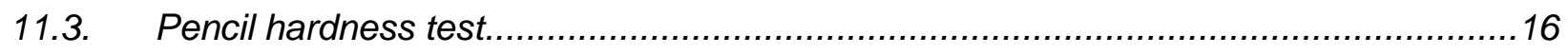

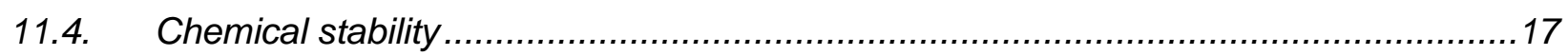

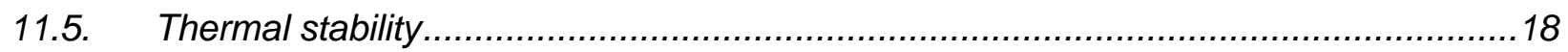

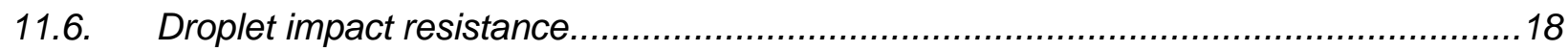

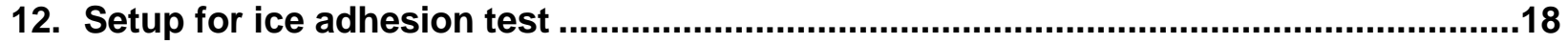

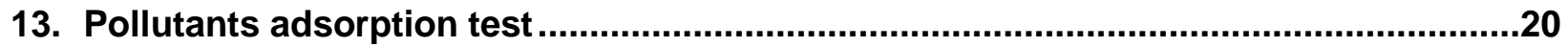

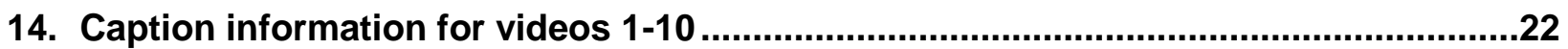

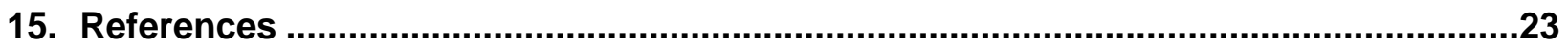

\section{Materials}

Microscopic glass slides $(75 \mathrm{~mm} \times 25 \mathrm{~mm})$ were purchased from Thorlabs. All the chemicals including, zirconium chloride octahydrate $\left(\mathrm{ZrCl}_{2} .8 \mathrm{H}_{2} \mathrm{O}\right)$, dihydroxyterepthalic acid (DHTPA), dimethyl formamide, glycol, glycerol, ethanol, acetone, isopropanol, 1butanol, n-hexane, butanone, 1,2-butanediol, decanol, cyclohexaneol, benzene, toluene, chloroform, dimethoxy methyl silane, 3-aminopropyltriethoxy silane (APTES), trichlorohexyl silane, trichlorododecyl silane, trichlorooctadecyl silane (OTS), rhodamine $B$, sodium hydroxide $(\mathrm{NaOH})$, sulphuric acid $\left(\mathrm{H}_{2} \mathrm{SO}_{4}\right)$, hydrochloric acid $(\mathrm{HCl})$ and silicon oil $(500 \mathrm{cSt}$ ) were purchased from Sigma Aldrich. Commercial vegetable oil was purchased from a local supermarket (Tesco). All the chemicals were used without further purification.

\section{Instrumentation}

The surfaces morphologies were imaged using scanning electron microscopy (SEM) (Carl Zeiss EVO25) and atomic force microscopy (AFM) (Bruker ICON SPM). For SEM, the specimens were immobilized on a metal stub with double-sided adhesive carbon tape and coated with a thin gold film, then observed at $20 \mathrm{kV}$ voltage and $10 \mathrm{pA}$ current. The MOF coated glass was subjected to Raman spectroscopy using a Renishaw confocal microscopy in the region of 100 to $2000 \mathrm{~cm}^{-1}$ to confirm their chemical structures. The PXRD of the MOFs powder was recorded on Stoe STADI-P spectrometer at ambient temperature, with tube voltage of $40 \mathrm{kV}$, tube current of $40 \mathrm{~mA}$ in a stepwise scan mode $\left(5^{\circ} \mathrm{min}^{-1}\right)$. FTIR spectra was recorded with Perkin Elmer Spectrum Two ${ }^{\mathrm{TM}}$ spectrophotometer in the region of 600 to $4000 \mathrm{~cm}^{-1}$. Transmission electron microscopy (TEM) micrographs were collected using a JEOL JEM2100 microscope at a beam acceleration field of $200 \mathrm{kV}$. Ellipsometry data was collected using Semilab SE-2000 at 
room temperature using a wavelength range of $240 \mathrm{~nm}-2000 \mathrm{~nm}$. The data was modelled using Tauc-Lorentz equation in SEMILAB software.

\section{MOF film fabrication on glass}

3.1. APTES functionalization: The substrate (glass) was pre-functionalized with APTES to obtain firmly attached and uniform MOF film. In brief, a glass slide was immersed into $1 \% \mathrm{n}$-hexane solution of APTES in a petri dish and incubated for $2 \mathrm{~h}$ at room temperature. The slide was thoroughly rinsed with $\mathrm{n}$-hexane to remove the physiosorbed APTES molecules and then dried under $\mathrm{N}_{2}$.

3.2. Layer-by-layer growth of MOFs: The synthesis parameters including number and time for each repeated cycle, reagents concentration and washing step were optimized for L/L growth of Zr-MOFs (see also the next section). In a typical optimal cycle of L/L growth of MOF, glass slides pre-functionalized with APTES were immersed into $100 \mathrm{~mL}$ of DMF solution of DHTPA $(25 \mathrm{mM})$ in a tightly closed glass bottle for $4 \mathrm{hrs}$ to get uniform self-assembly of DHTPA onto the substrate at $120^{\circ} \mathrm{C}$. The substrate was then rinsed in DMF and immersed in $25 \mathrm{mM}$ DMF solution of $\mathrm{ZrCl} 2.8 \mathrm{H}_{2} \mathrm{O}$ for 20 minutes at $120^{\circ} \mathrm{C}$ followed by washing (which included 1-minute sonication) and immersion into DHTPA solution for another 20 minutes. This completed one cycle of MOF growth. Various number of cycles were repeated to optimize and achieve the controlled growth of the MOF films. Finally, the surface was thoroughly washed by sonicating in DMF to remove metal or linker aggregates. The MOF on glass was then treated in chloroform for $48 \mathrm{hrs}$ to activate the pores (to remove the traces of DMF and any other solvents) and then vacuum dried overnight at $100{ }^{\circ} \mathrm{C}$. After synthesis, the MOF film was characterized to determine crystallinity using PXRD (Fig. S1), chemical structure by Raman (Fig. S2) and FTIR (Fig. S3) and elemental distribution with EDS mapping (Fig. S4). 


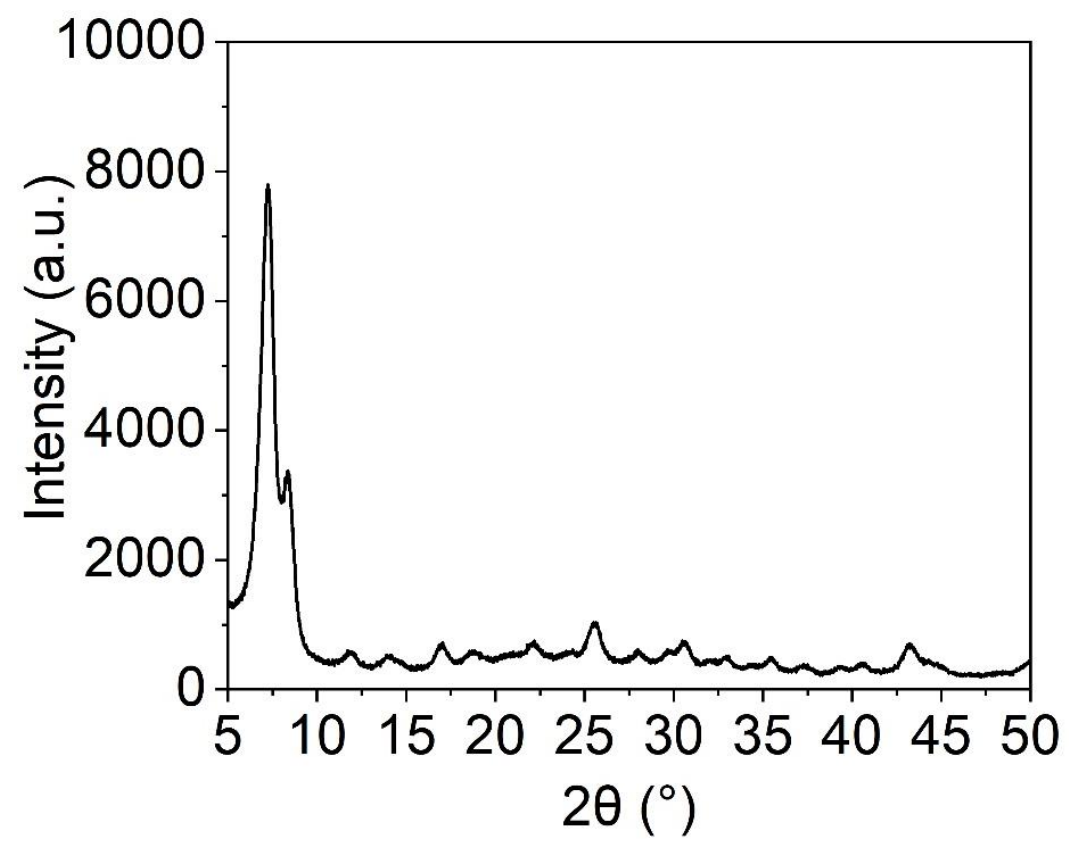

Figure S1. The PXRD graph of MOF film ${ }^{1}$

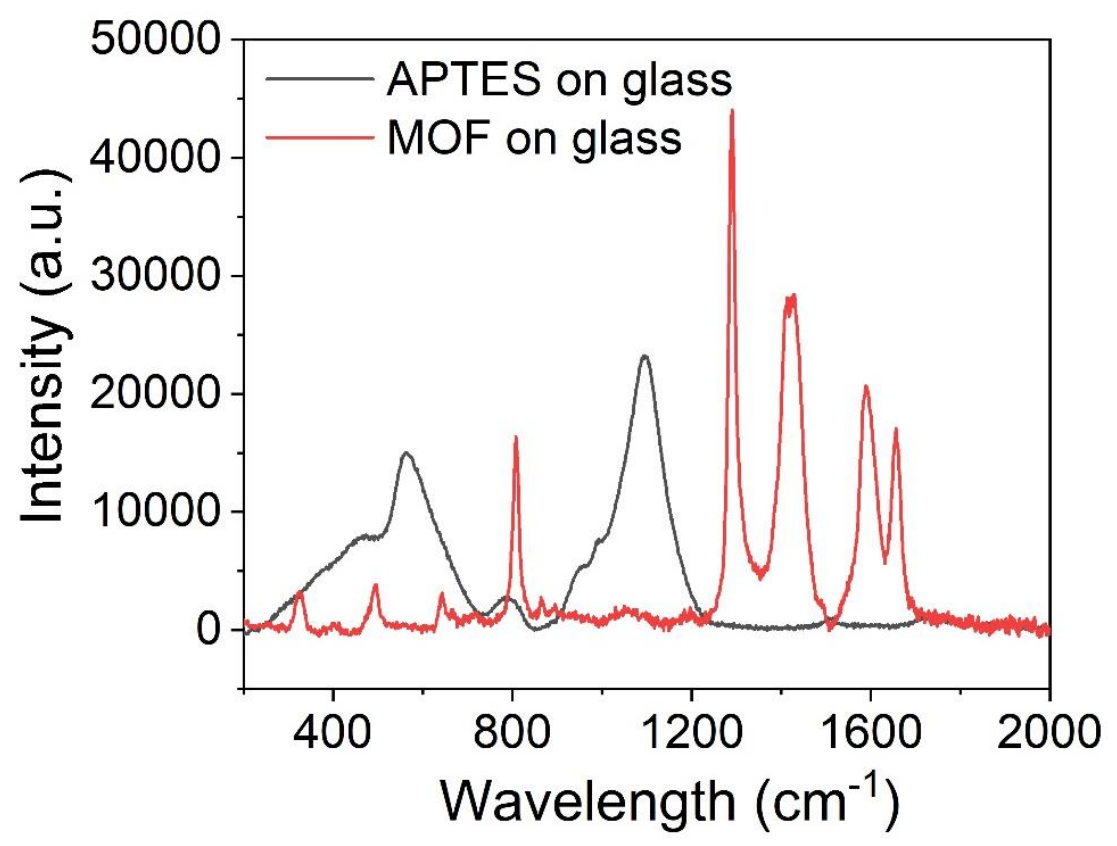

Figure S2. Raman spectrum of glass functionalized with APTES and after MOFs growth confirming the successful functionalization of APTES functionalisation and chemical structure of the hydroxylated Zr-MOFs, in agreement with the previously published reports ${ }^{2}$. 


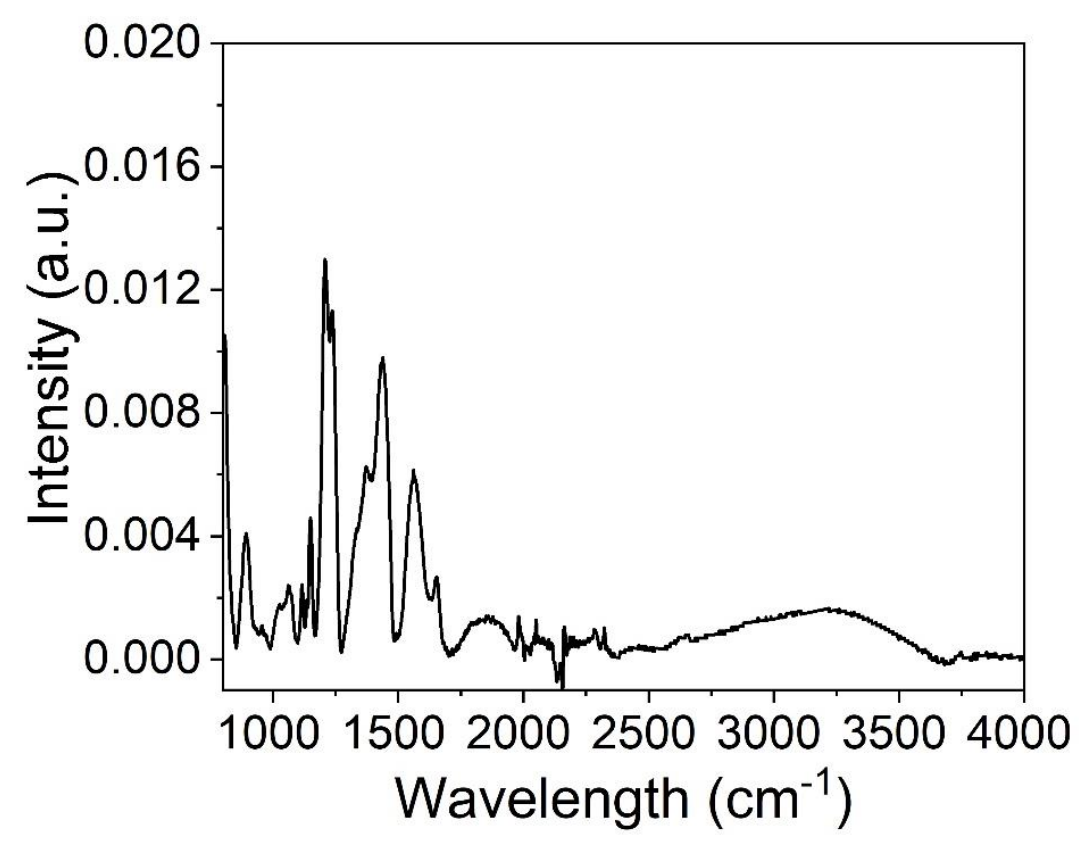

Figure S3. FTIR spectra of MOF further confirming the chemical structure. The shark peaks at $1435 \mathrm{~cm}^{-1}$ and $1563 \mathrm{~cm}^{-1}$ are ascribed to the in and out-of phase stretching modes of the carboxylate group presented in the linker. The broad peak for hydroxyl groups is observed at $3200-3500 \mathrm{~cm}^{-1}$. 

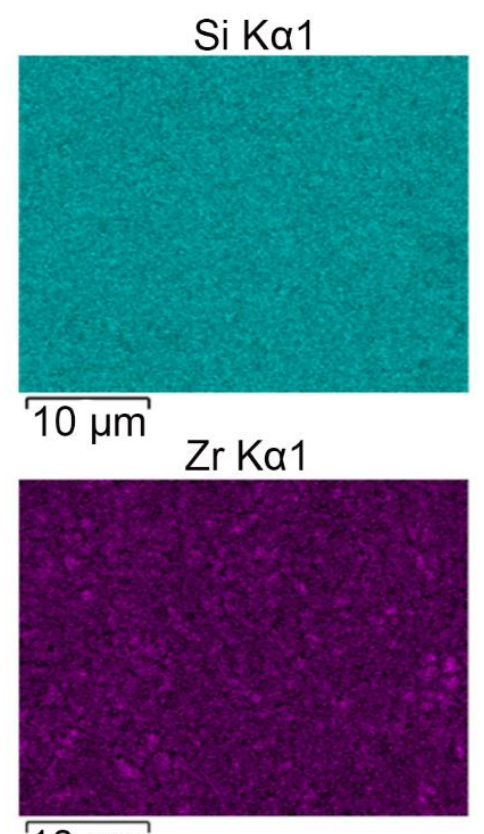

$10 \mu \mathrm{m}$
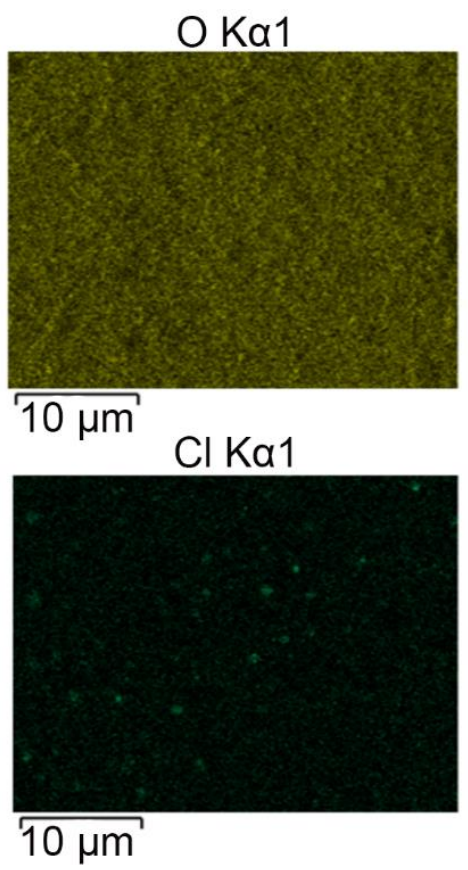

Figure S4. Elemental mapping of the hydrophobic MOF surface modified with alkyl silane, showing the distribution of $\mathrm{Zr}$ and other major representative elements.

3.3. Synthesis optimization of the MOF film: Obtaining a covalently attached MOF film with controlled roughness on a glass substrate was a crucial step in this study. L/L technique to grow MOFs epitaxially offer a means to grow films with controlled thickness and has been used for many applications such as sensors, molecular devices and catalysis etc ${ }^{3}$. However, for the amphiphobic surfaces developed in this work, controlling roughness was also critical and was achieved through rigorous synthesis optimization, which involved tuning three parameters: concentration of reagents, number of cycles and washing steps. The film growth was tracked using SEM imaging to check the change in the morphology after each layer as shown in Fig. S5. For the concentration of organic linker and metal, 25 $\mathrm{mM}$ found to be the optimum and yielded a uniform MOF film with controlled roughness (Fig. $1 \mathrm{~F}$ and $1 \mathrm{G}$ ). The growth of MOF film was recorded to be very slow at lower concentration (5 and $10 \mathrm{mM}$ ) of reagents as shown in Fig. S6A and resulted in nanometre sized but uniformly distributed particles. However, the obtained roughness was very low even after $10-15$ repeated cycles. Conversely, with $50 \mathrm{mM}$ concentration, the growth of larger MOF clusters were observed (Fig. S6B) even after the $3^{\text {rd }}$ layer unlike the optimized film where a uniform film was formed first and then the particles started emerging afterwards (Fig. 3D). The washing procedure in between the growth cycles was recorded to be very important in maintaining transparency as it was necessary to remove the metal 
aggregates and physically adsorbed linkers. In addition to the simple rinsing, we introduced the 60 s sonication after each cycle to ensure the removal of unreacted reagents.

3.4. Effect of APTES functionalization: The role of APTES was also investigated; the growth of MOF without APTES was very slow and resulted into non-uniform MOF bulges as shown in Fig. S6C. Whereas, APTES functionalized glass produced uniform and controlled MOF film as shown in Fig. 1E-G in the main manuscript.

3.5. Post-synthesis functionalization of MOFs: Alkyl silanes were used to convert the as-synthesized hydrophilic MOF film into hydrophobic structures. Liquid immersion method was adopted as used for APTES functionalization. The glass substrate with MOF film was immersed into $1 \%$ solution of silanes in $n$-hexane (trichlorohexyl silane, trichlorododecyl silane, trichlorooctadecyl silane) for $2 \mathrm{hrs}$ and placed at $120{ }^{\circ} \mathrm{C}$ for another $2 \mathrm{hrs}$ then rinsed with $\mathrm{n}$-hexane. The hydrophobic substrate was then dried under $\mathrm{N}_{2}$ stream and stored for further characterization.
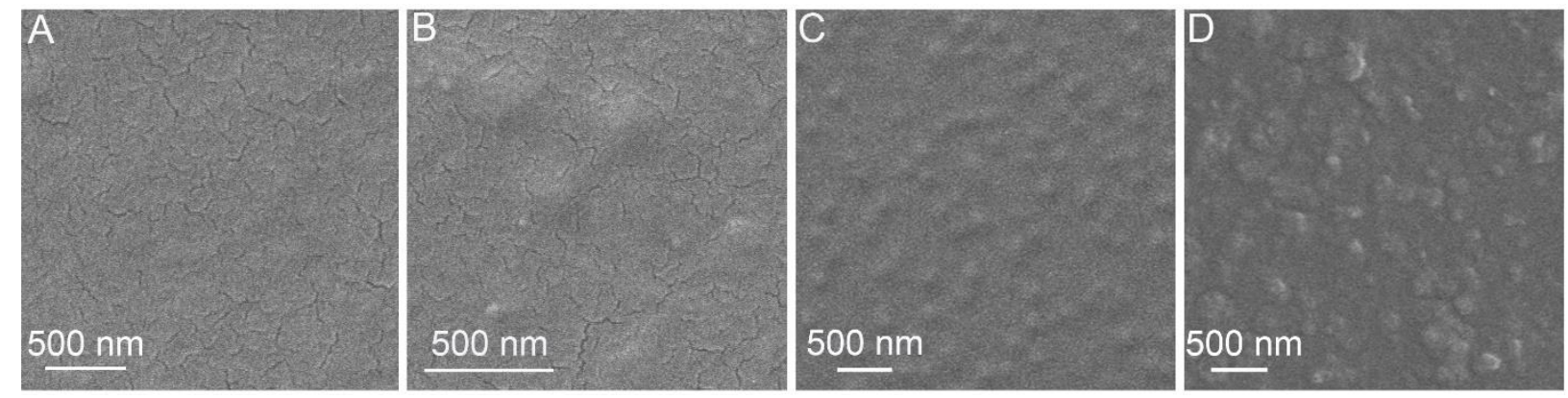

Figure S5. SEM micrographs tracking MOF growth with increasing layers (A) After first layer, the growth is even with numerous cracks. (B) The image showing MOF structures after second layer where the cracks started to disappear, and MOF particles start to grow as bulges. (C) The cracks completely disappeared after 4 layers with several MOF particles clearly emerging which turned into rough particles after (D) 5th layer.
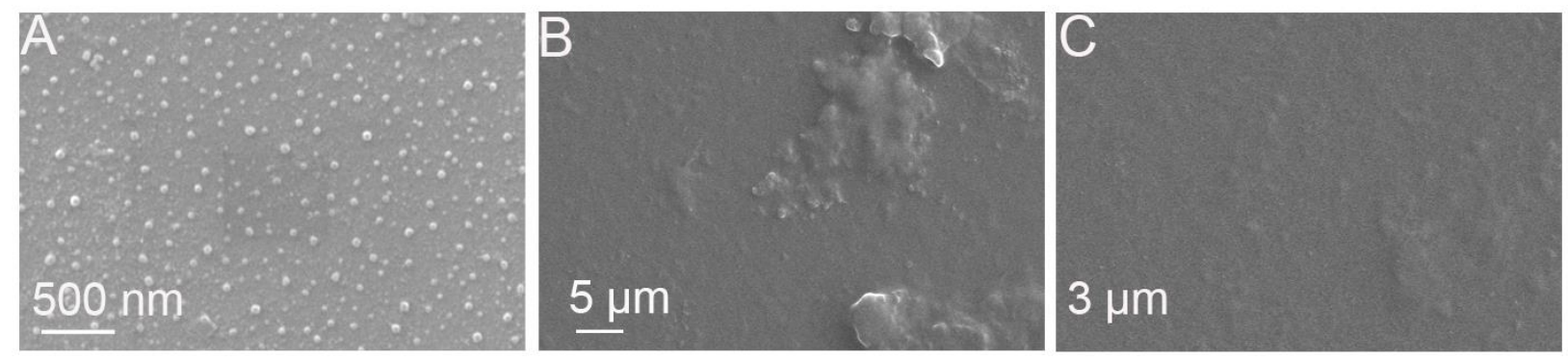

Figure S6. Morphology characterization of MOF film at different concentration of organic linker and metal source for synthesis optimization. SEM image MOF films (A) at low (10 $\mathrm{mM}$ ) concentration of organic linker and metal, recorded after 10 cycles, and (B) at 50 
$\mathrm{mM}$ concentration, after 4 cycles and (C) after 10 cycles, grown without APTES functionalization

\section{Fabrication of comparative surfaces}

4.1. Liquid infused MOFs for comparison: The MOF SLIPS was prepared using simple procedure by replacing silane with silicon oil (500 cSt). SLIPS surfaces are now well established for low hysteresis and droplet sliding despite lower overall contact angles compared to superamphiphobic surfaces (with $\theta_{A}>150^{\circ}$ ). The MOF coated glass slide was placed horizontally and infused with $2 \mathrm{~mL}$ silicon oil for $24 \mathrm{hrs}$ and tilted to $90^{\circ}$ for $2 \mathrm{hrs}$ to get rid of excess oil. The MOF SLIPS was used as a benchmark for our MOF on glass surfaces and to establish their superiority in terms of drop sliding speed and stability under continuous water flow.

4.2. Fabrication of slippery omniphobic (SOCAL) coating: A SOCAL surface was prepared to compare for ice adhesion strength with our nanohierarchical MOF surface. The fabrication process was adopted from previously published work ${ }^{4}$. In brief, a mixture of isopropanol (50 g), dimethyldimethoxysilane $(5 \mathrm{~g})$ and sulfuric acid $(1 \mathrm{~g})$ was prepared in a clean glass bottle. The solution was sonicated for 120 seconds and stored at room temperature for further use. An oxygen plasma cleaned glass slide was submerged in the solution for $5-10$ seconds and withdrawn gradually. The excess liquid was drained from the surface. The substrate was dried at room temperature ( $>60-70 \%$ relative humidity) for $1 \mathrm{hr}$. Then the surface was rinsed with hexane, toluene and isopropanol and dried under $\mathrm{N}_{2}$.

4.3. Fabrication of OTS coating: An OTS coating was prepared by liquid immersion method. In brief, a glass slide was immersed into $1 \%$ of $n$-hexane solution of OTS in a petri dish and incubated for $2 \mathrm{~h}$ at room temperature. The slide was thoroughly rinsed with $\mathrm{n}$-hexane to remove the physiosorbed silane molecules and then dried under $\mathrm{N}_{2}$.

\section{Wettability tests}

A custom goniometer setup was used for contact angles measurements ${ }^{5}$. The setup consists of an adjustable stage, retort stand and syringe pump (World Precision Instruments, Aladdin single-syringe infusion pump), a light source (Thorlabs, OSL2) and a zoom lens (Thorlabs, MVL7000) fitted to a CMOS camera. To measure the contact angles and the contact angle hysteresis, the videos of the droplet were analysed with MATLAB. 


\section{Solid fraction of the MOF surface}

The solid fraction, $\phi$, of MOF was estimated, by considering the geometry of the MOF pores and the corresponding unit cell (see Fig. S7), to be

$$
\phi=1-\frac{S_{\text {hole }}}{S_{\text {cell }}}=1-\frac{\pi R^{2}}{3 \sqrt{3} R^{2}}=0.4
$$

where $S_{\text {cell }}$ and $S_{\text {hole }}$ denote the surface area of the unit cell and the hole inside cell, respectively. $R$ is the radius of the hole (approximated to be a spherical). The reported geometrical details and molecular dimensions (pore diameter $\sim 6 \AA$ ) from literature ${ }^{6,7}$ were used to sketch the unit cell in Fig. S7.

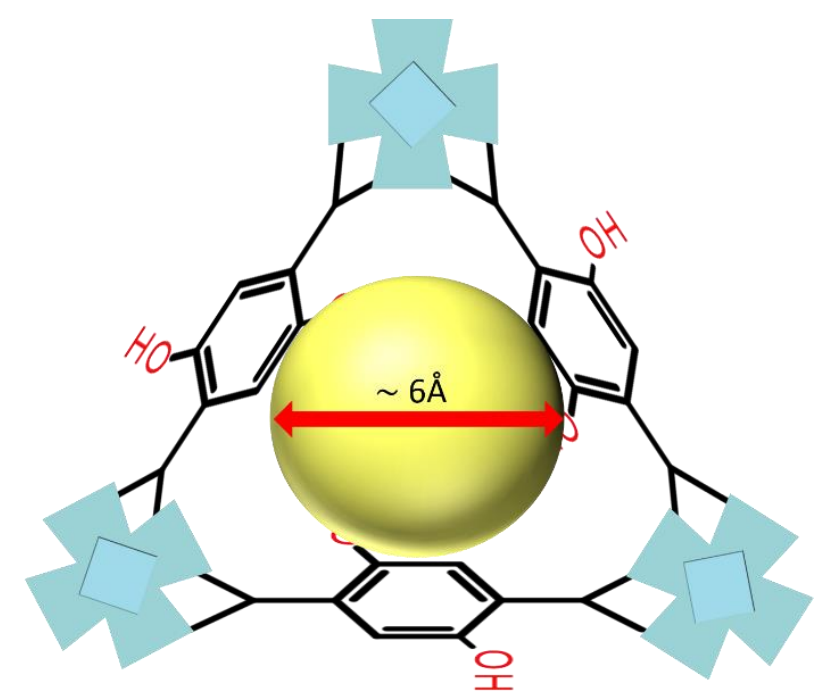

Figure S7. schematic representation of the pore cavity of UIO-66 used for the solid fraction calculation.

\section{Drop mobility tests}

Drop mobility test was carried by releasing water droplets of various volumes $(5,10,15$, $20,25,30,35$, and $40 \mu \mathrm{L}$ ) on the MOF on glass surface lying on a $30^{\circ}$ tilted stage. The average speed was obtained by recording thrice using high-speed camera (Phantom V411). The same process was following for MOF SLIPS and for thermal stability tests where the droplet of $20 \mu \mathrm{L}$ was used after heating the surface at specific temperature for $1 \mathrm{hr}$. 


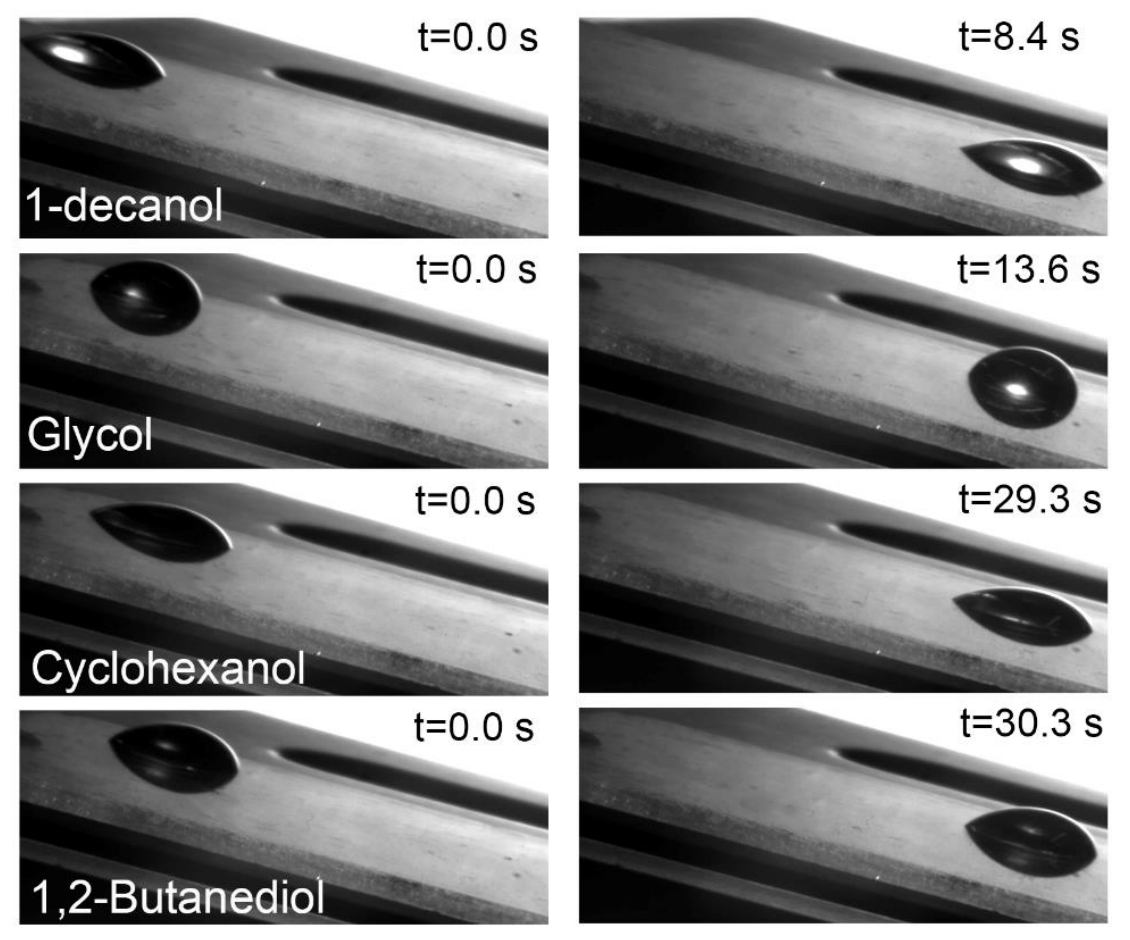

Figure S8. Image sequence showing the sliding of low surface tension liquids at $15^{\circ}$ tilt angles on nanohierachical MOF surface.

\section{Transparency tuning and measurement}

Transparency of the MOF coated glass surface was tuned by controlling the thickness/number of MOFs layers. As shown in Fig. 2E, no change in the transmittance recorded up to 6 layers of MOFs. However, the transmittance decreased to $\sim 90 \%$ from $>93 \%$ when the number of layers exceeded six which could also be observed from the photographs presented in Fig. S9. The successive change in the transmittance with increase in number of MOF layers was also visible to the naked eye (Fig. S9). The surface roughness is also increased with the number of layers and thus, the $\Delta \theta$ started increasing after 6 layers, possibly due to the adverse effect of roughness on slippery behaviour. This is understandably given the fact that beyond a critical roughness, the solid liquid contact will raise the liquid adhesion to the films. The scattering behaviour of the films was measured in transmission using a Radiant Zemax Imaging Sphere for Scatter and Appearance Measurement. The samples were illuminated at a normal angle of incidence at $50 \mathrm{~nm}$ wavelength intervals between 400 and $700 \mathrm{~nm}$ wavelengths. To get the single transmittance value at photopic response, the photopic response function was calculated using MATLAB. 


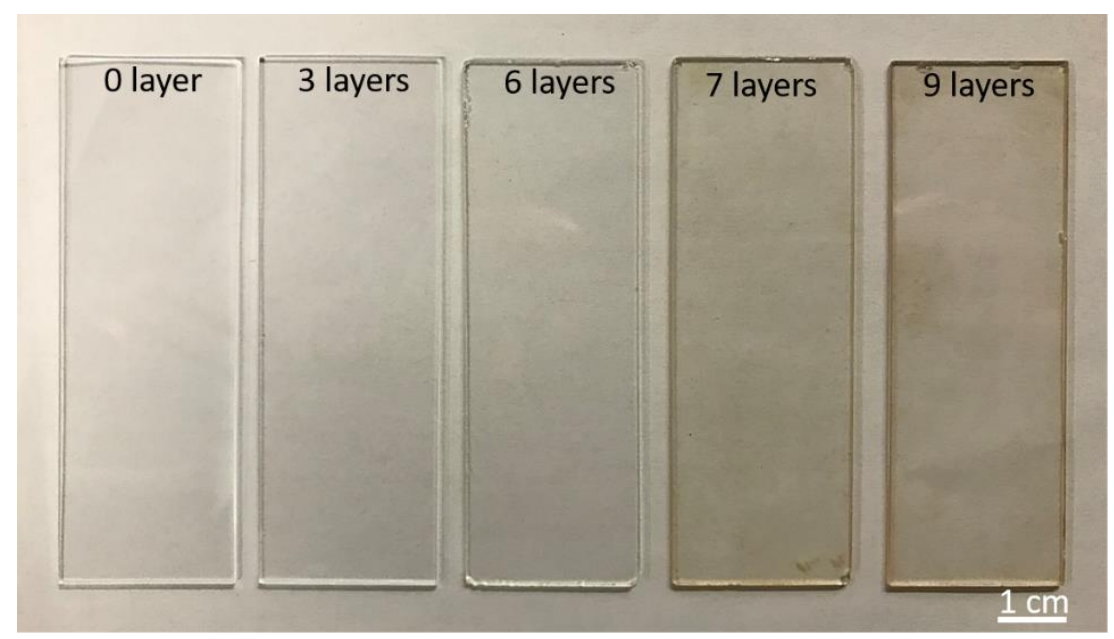

Figure S9. Photograph showing the visual change in transparency of glass slides with the increasing number of MOF layers.

\section{Theoretical droplet sliding velocity calculations}

Theoretical velocity of the droplets is calculated through force balance along the contact line in the sliding direction ${ }^{8}$. We observed that the droplet moves at a steady velocity, hence the transient term of the velocity $d U / d t$ is zero. The effect of air drag on the droplet is neglected.

The adhesion force is $F_{a d} \sim \gamma D\left(\cos \theta_{A}-\cos \theta_{R}\right)$ and the viscous shear force scales as $F_{\tau} \sim \mu U R$. The symbol $D$ denotes the droplet diameter prior to deformation (the same area as the ellipse), $R$ is the droplet radius, $\mu$ is the liquid viscosity, $\theta_{R}$ is the receding (uphill) and $\theta_{A}$ the advancing (downhill) contact angle, respectively. The adhesion and viscous forces act jointly against the gravitation force along the inclined substrate, which favours droplet acceleration. By balancing these forces, the theoretical velocity of the droplet is obtained as

$$
U=K \frac{\left(m g \sin \delta-\gamma D\left(\cos \theta_{A}-\cos \theta_{R}\right)\right)}{\mu R}
$$

where from ref. ${ }^{5} \mathrm{~K}=0.011$ and $\delta$ is the substrate inclination, $m$ the droplet mass and $g$ the acceleration due to gravity.

\section{Setup for high-speed jet impact}


To overcome the limitation of low terminal speed of free-falling droplets, a setup shown in Fig. S10 was used to obtain a continuous and controlled water jet ${ }^{5}$. A high-pressure nitrogen gas cylinder connected to an electronic pressure valve was used to force water through a nozzle (a needle/syringe assembly). The water jet diameter was $0.5 \mathrm{~mm}$ and $2.5 \mathrm{~mm}$. Due to system transients, upon application of pressure control signal on the electronic control valve, the gas back pressure on the piston in the syringe will ramp up to the maximum $\sim 13$ bar over a finite time period. This transient process should lead to a time dependent rise in jet speed before levelling off to a steady value corresponding to the maximum applied pressure. To unravel this transience, we recorded the motion of the piston/water interface inside the syringe during typical jet impact process using the highspeed camera. The motion of the piston could be used to determine the jet speeds through simple mass conservation and knowledge of the cylinder and the nozzle diameters. Thus, after reaching steady speed, if in time $\Delta t$ the piston in the syringe moves by a distance $\Delta \mathrm{h}$, we can write $\pi d_{s}^{2} \Delta h / 4=\pi d_{n}^{2} \mathrm{~V} \Delta t / 4$, where $d_{s}$ is the cylinder diameter, $d_{n}$ the nozzle (jet) diameter and $V$ the jet speed. The maximum jet speed reached in our experiments $(35.1 \mathrm{~m} / \mathrm{s})$ was determined by averaging the maximum speeds in different tests. The corresponding liquid Weber number $\left(W e_{l}=\frac{\rho V^{2} d_{n}}{\gamma}\right)$ for the $0.5 \mathrm{~mm}$ and $2.5 \mathrm{~mm}$ jet are $\sim 8,500$ and $\sim 42,500$ (supplementary movie 5 and 6 ). The surface showed no signs of liquid impalement even after repeated jet at least 3 times on same spot. The lack of liquid impalement was assessed using droplet mobility test. This comprised of positioning the surfaces horizontally and placing a droplet at the centre of impact location (Fig. S13 and supplementary movie 8). Then the surface was tilted gently to ensure that the droplet slid off, confirming a lack of liquid impalement during jet impact.

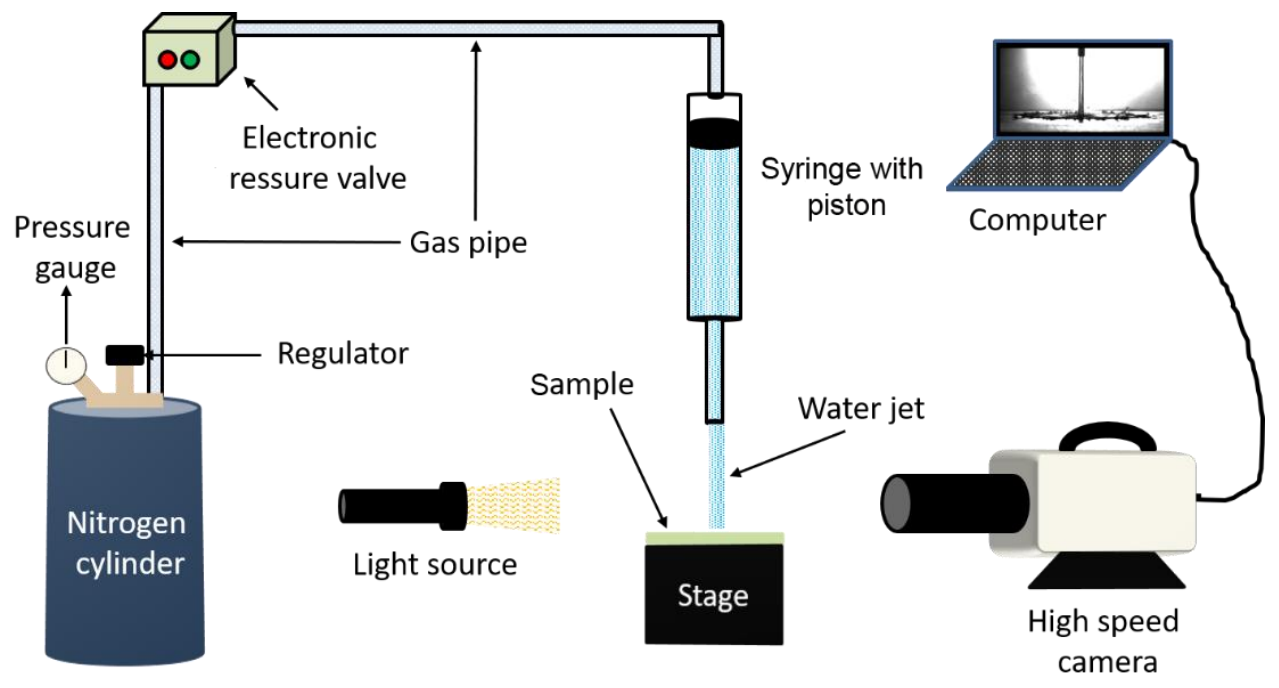

Figure S10. Diagrammatic representation of water jet impact experiments. 


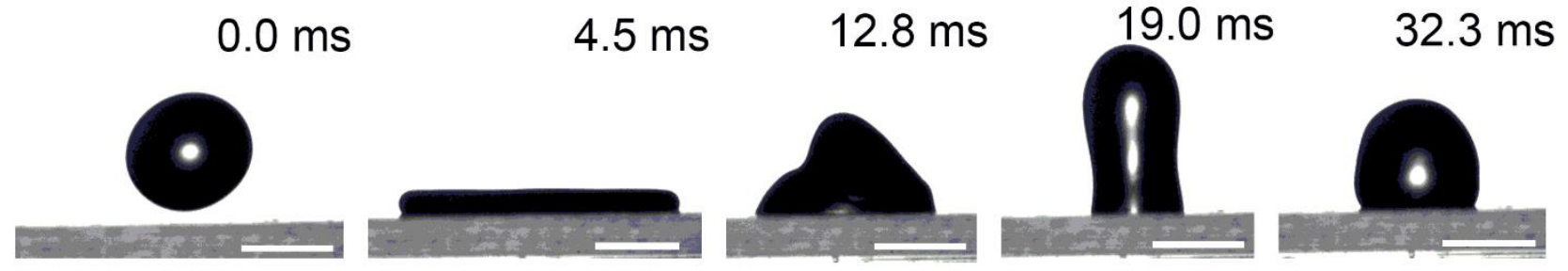

Figure S11. Time-lapse snapshots of drop impact on silanized glass surfaces taken as a control. The surface showed no bouncing even at low velocity of $1.2 \mathrm{~m} / \mathrm{s}$ (c.f. Fig. $3 \mathrm{~A}$ and supplementary Movie 4).

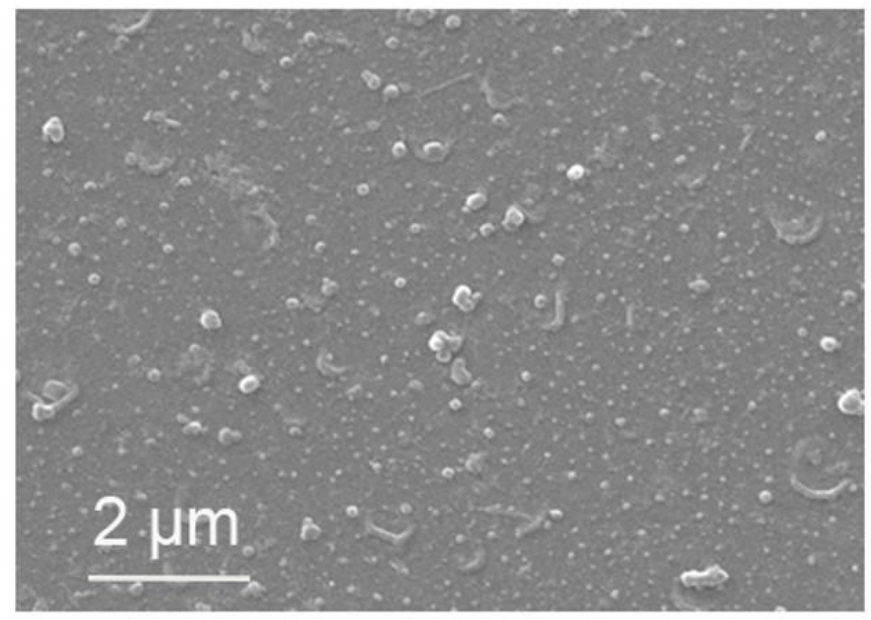

Figure S12. SEM image showing morphology of MOF-on-glass surface after repeated jet impact test. 

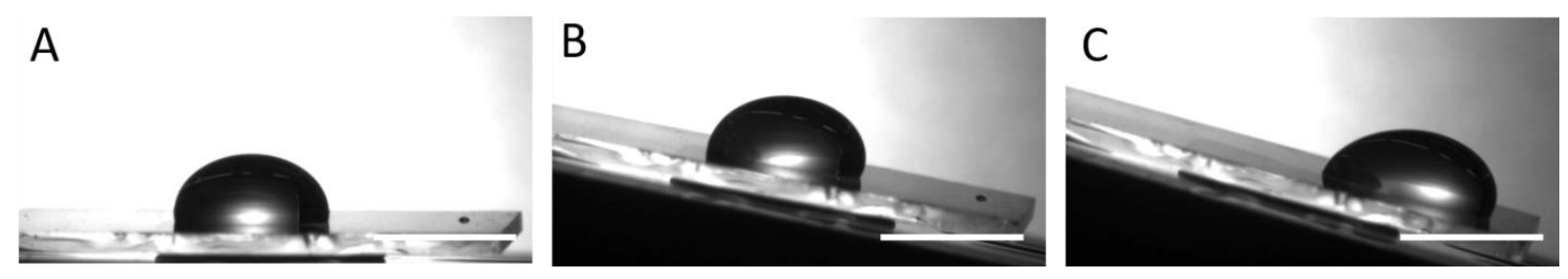

Figure S13. A series of snapshots showing free sliding of droplet on a coated surface after jet impact test. (A) A water droplet was gently placed at the area repeatedly impacted by high-speed jet at $35 \mathrm{~m} / \mathrm{s}$ and tilted slowly ( $B$ and $C$ ) to confirm lack of any pinning and maintenance of slippery behaviour of the surface. Scale bar is $5 \mathrm{~mm}$.

\section{Stability assessment}

11.1. Hydrophobicity retention test: Slippery behaviour and hydrophobicity (liquid repellence) of the SLIPS surfaces are known to be susceptible to gradual drainage of the lubricant film under shear flow or sustained sliding of droplets on them. Our surfaces, lacking any oil infusion, are expected be immune to this. For this test, a bespoke setup comprising a water tank (plastic, 2-L water bag) connected to syringe via flow controller was used (Fig. S14). A continuous flow of water droplets (at the rate of $1 \mathrm{~L} / \mathrm{h}$ from $2 \mathrm{~cm}$ height) dripped onto test surface placed on a $30^{\circ}$ titled stage. This created a continuous stream of drops sliding off the surfaces under test. Contact angle measurements were takes after drainage of $50 \mathrm{~mL}, 100$ $\mathrm{mL}, 500 \mathrm{~mL}, 1,000 \mathrm{~mL}, 2,000 \mathrm{~mL}, 3,000 \mathrm{~mL}, 4,000 \mathrm{~mL}$ and $5,000 \mathrm{~mL}$ of water from the bag. The recorded contact angle hysteresis values are plotted in Fig. $2 \mathrm{G}$. 


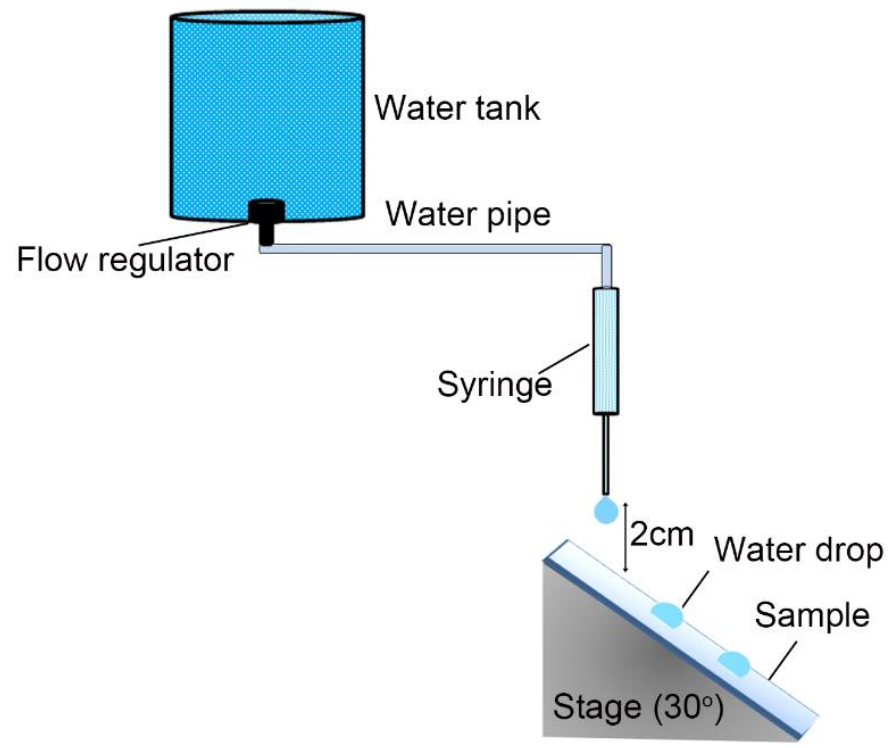

Figure S14. Schematic of the setup used to test the retention of hydrophobicity. Both nanohierarchical MOF surface and the corresponding MOF based SLIPS were tested with same protocol.

11.2. Mechanical stability: To evaluate the mechanical durability of the coatings, a pressure-sensitive and strong adhesive tape (3M VHB ${ }^{\mathrm{TM}}$ tape 5952 with adhesive peel strength of $3,900 \mathrm{~N} / \mathrm{m}$ ) was used. The tape was applied on horizontally placed hydrophobic MOF surface by rolling 2-kg steel roller on the tape and then the tape was removed after waiting for $60 \mathrm{~s}$ as shown in Fig. S15. This whole process was considered as one cycle; the contact angle measurements were performed after each cycle. The process was repeated, and a fresh piece of tape was used in each cycle.
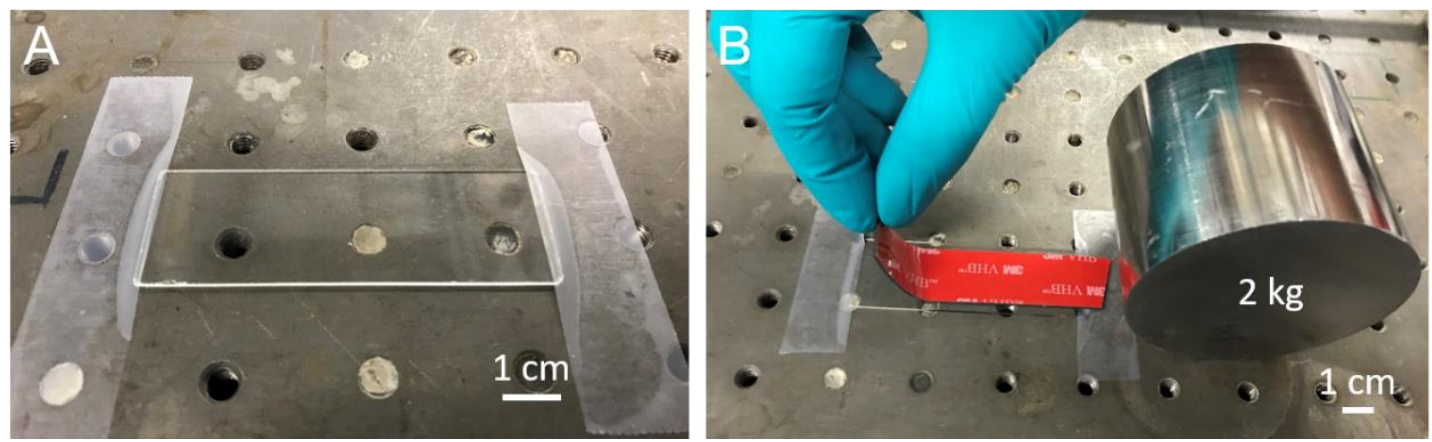

Figure S15. Tape peel test to check the adhesion of MOF layer to the glass. (A) A MOF coated glass slide fixed with transparent tape and (B) application of strong adhesive tape on MOF on glass substrate. 


\subsection{Pencil hardness test:}

The resistance of MOF films to scratches and wear was tested via standard pencil hardness test (ASTM D 3363). Pencils with five different hardness level, $\mathrm{HB}, 2 \mathrm{H}, 3 \mathrm{H}, 4 \mathrm{H}$ and $5 \mathrm{H}$ were used in the test and the details are shown in Fig. S16. The pencil marks could be clearly observed on surface (Fig. S16A) just after the test. However, the scratches completely disappeared after gentle cleaning with the tissue paper (Fig. S16B), suggesting that there was no permanent damage into the MOF films, rather the marks were left by pencil lead erosion. For these tests, pencil leads with flat cross-section were achieved (Fig. S16E) by rubbing the pencils on sand paper. The flat pencil leads were pushed horizontally laid MOF surface at $\sim 45^{\circ}$ angle (Fig. S16C and S16D). After the test, integrity of the surface was checked by measuring the contact angles and drop mobility on the scratched areas. The advancing $\left(113^{\circ} \pm 2\right)$, receding $\left(102^{\circ} \pm 2\right)$ and hysteresis $\left(10^{\circ} \pm 1\right)$ angles all remained unchanged. No pinning was observed in drop mobility test (see the supplementary movie 10), confirming the surface integrity and excellent resistance against mechanical scratches.

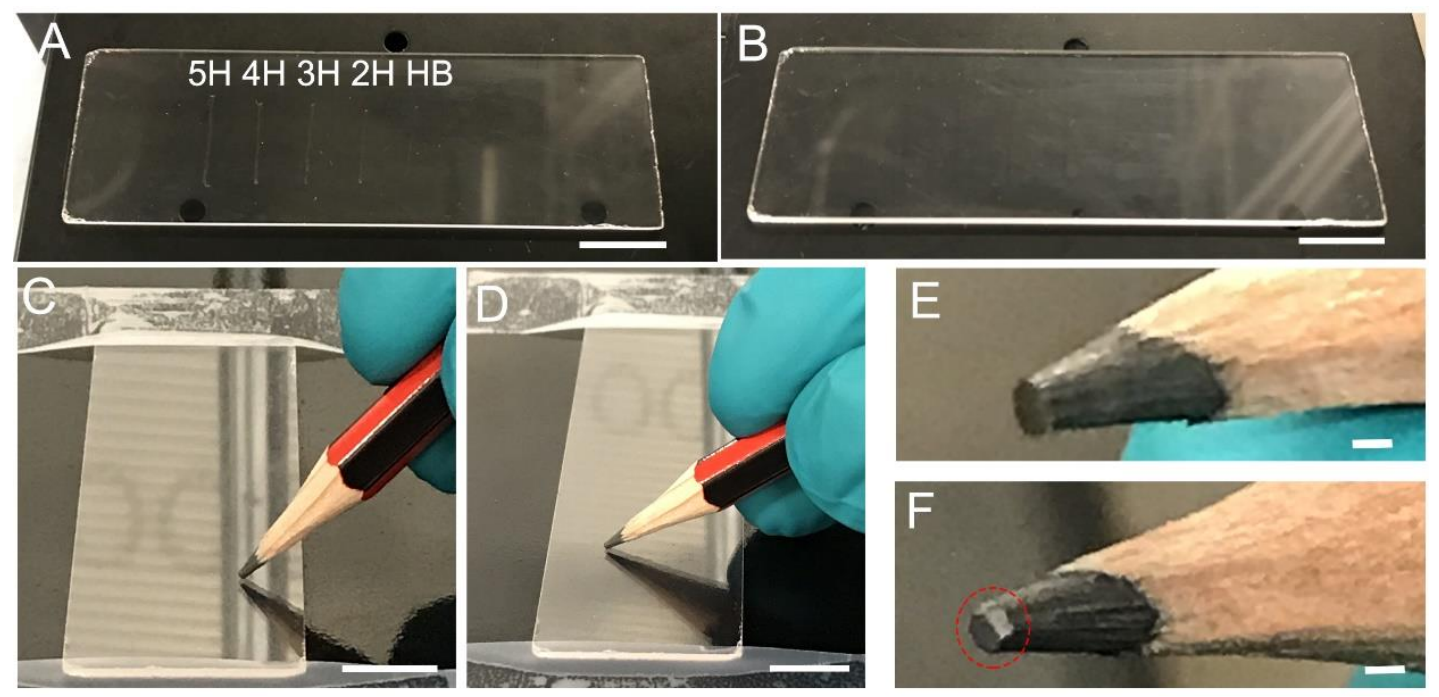

Figure S16. Pencil hardness test. (A) The nanohierarchical MOF surface showing marks left by standard scratch test performed using pencils of different hardness and (B) the pencil marks disappeared after gentle cleaning of the surface with tissue paper. (C-D) A full cycle of pushing the pencil at $\sim 45^{\circ}$ on the MOF surface and (E) the close photograph of flattened pencil lead before and (F) after test. Scale bar is $1 \mathrm{~cm}(A-D)$ and $1 \mathrm{~mm}(E-F)$. 


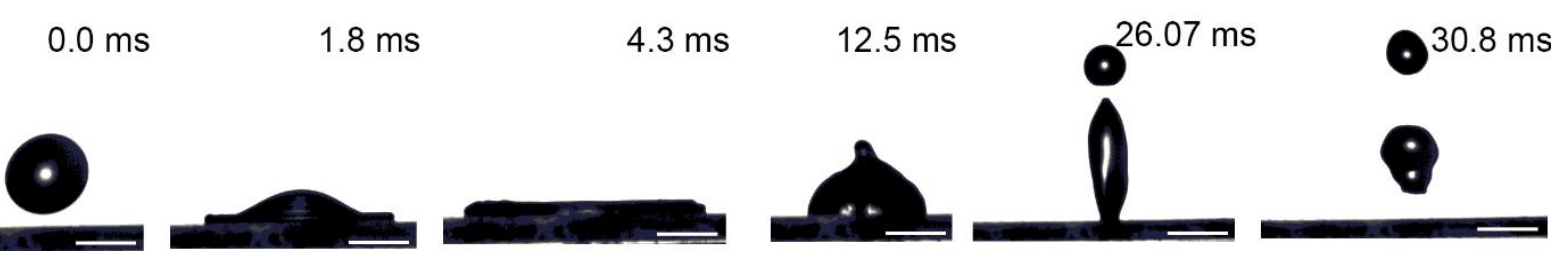

Figure S17. Time-lapse snapshots from drop impact test $(1.2 \mathrm{~m} / \mathrm{s})$ on a nanohierarchical MOF surface after 50 tape peel cycles. Scale bar is $2 \mathrm{~mm}$.

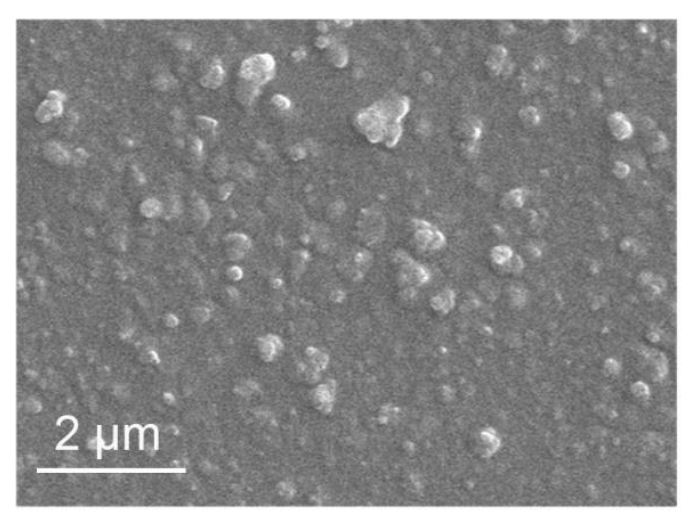

Figure S18. SEM micrograph of the MOF surface after 50 repeated tape peel cycles.

11.4. Chemical stability: The chemical stability test was performed in acidic $(\mathrm{pH}, 1-2)$ and basic $(\mathrm{pH}, 11-12)$ solutions. The test surfaces were immersed for specific time periods, rinsed in DI water and dried followed by contact angle measurements.
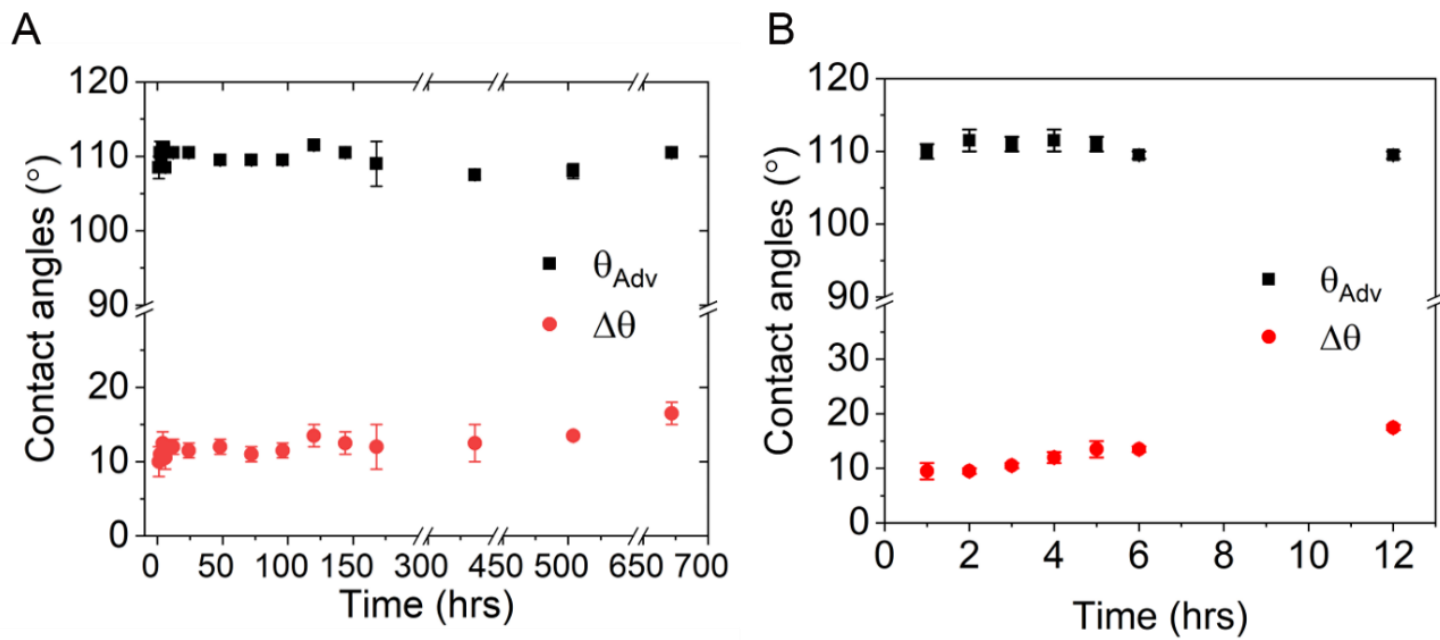

Figure S19. Acid-base stability. (A) Effect of immersion in acidic $\mathrm{pH}(1-2)$, the non-wetting features are maintained for 1 month, when hysteresis slightly increased from $10^{\circ}$ to $15^{\circ}$. 
(B) The base stability graph showing the change in contact angles ( $\theta \_A$ and $\Delta \theta$ ) on MOF on glass surface. The surface was immersed into basic solution of $\mathrm{pH}=11-12$ and subjected to contact angle measurement at predetermined time intervals.

11.5. Thermal stability: To evaluate the thermal stability of the MOF surface, the substrate was placed on a temperature-controlled hotplate at specific temperatures from $40^{\circ} \mathrm{C}$ to $200^{\circ} \mathrm{C}$ for $1 \mathrm{hr}$. The surface was brought to the room temperature and contact angles measurements and drop mobility tests were performed to determine the change in hysteresis angle and sliding speed.

11.6. Droplet impact resistance: For the droplet impact test, a high-speed camera (Phantom V411) was used to record at a rate of 10,000 frames per second. 2.7 $\mathrm{mm}$ size DI water droplets were released from various heights with the help of syringe pump (world precision instrument) connected to a syringe/needle assembly.

\section{Setup for ice adhesion test}

A custom designed bench-top icing chamber was used for ice adhesion experiments. The chamber comprised of a transparent, double-wall container and a cooling base (Fig. S20). The chamber has an external dimension of $(30 \mathrm{~cm} \times 20 \mathrm{~cm} \times 18 \mathrm{~cm})$ and a $10 \mathrm{~mm}$ air gap between its external and internal walls. Both walls are $5 \mathrm{~mm}$ thick and made of thermally insulating acrylic (Perspex) with a low thermal conductivity of $0.2 \mathrm{~W}(\mathrm{mK})^{-1}$. This air gap was evacuated to improve the insulation. Holes on walls were designed for introducing water into the measurement cuvettes and fitting in the metal rod used to deflect the cuvettes (see Fig. S20). As for the base, it consists of an aluminium frame, a base plate, a compact heat exchanger (P1805368, UK Exchangers), 4 axial fans below the heat exchanger (ARX CeraDyna Series, RS Components), a rotary aluminium stage, a plastic shaft and two Peltier cooling modules between the aluminium stage and the heat exchanger. The chamber temperature was measured using $4 \mathrm{~K}$-type thermocouples (HH506RA) and controlled using a refrigeration unit (FP50-HL Refrigerated/Heating Circulator, Julabo) with bath fluids ( $\mathrm{H} 5$, Julabo) connected to the compact heat exchanger. The chamber humidity was measured by a 3-pin humidity sensor $(\mathrm{HIH}-4000-$ 001, RS Components). The data acquisition system (DAQ) included a compact DAQ chassis (cDAQ-9174, National Instruments) with a temperature module (NI9213), a voltage module (NI-9263) and an analogue module (NI-9209) (75). During experiments, all openings/holes were blocked by putty-like adhesives (Blu Tack). For ice adhesion measurement, an extension rod connected to a force gauge (M4-50, MARK-10) were mounted on a custom-made driving system with a stepper motor (17HS19-2004S1). This 
enabled deflecting the cuvettes with frozen liquid laterally and measuring the required forces. The extension rod is $127 \mathrm{~mm}$ long, and its flattened end has a diameter of $25 \mathrm{~mm}$. LABVIEW software was used to operate and record the forces and determine the corresponding adhesion strength presented in Fig. 3F-G.

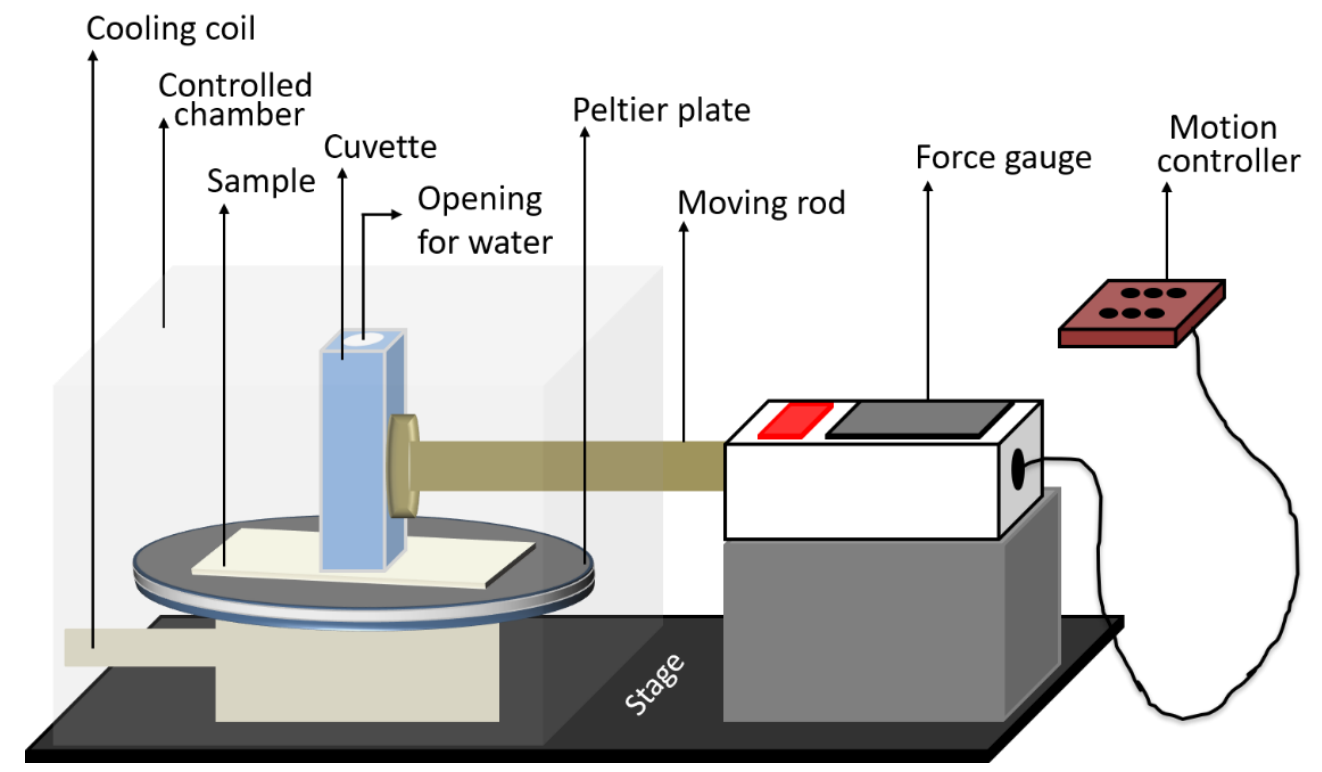

Figure S20. A schematic of the ice adhesion measurement setup. During each cycle tested, 3 cuvettes were placed on the glass slide (with or without MOF) under test and filled with water following by freezing at $-35^{\circ} \mathrm{C}$ for $1 \mathrm{hr}$ and then pushed with moving rod. The recorded forces were used to calculate the adhesion strength $(\mathrm{kPa})$ by diving the force with Cuvette cross-sectional area.

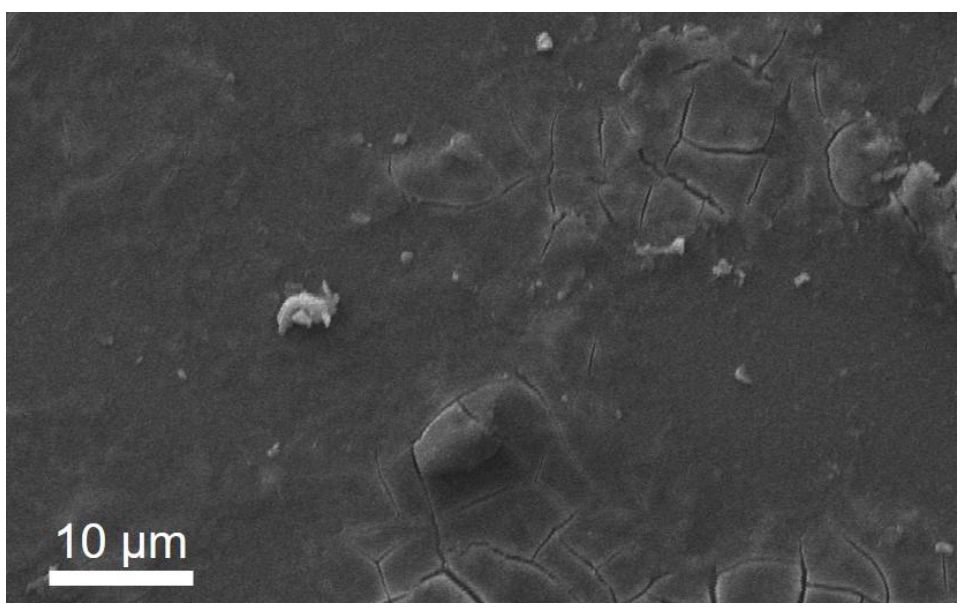

Figure S21 SEM image recorded after 15 icing-deicing cycles. 


\section{Pollutants adsorption test}

The MOFs used herein (UIO-66-OH) have well established ability to efficiently adsorb wide range of inorganic and organic pollutants ${ }^{9,10}$ and has been exploited to demonstrate pollution cleaning capability of MOF nanoparticles and MOF nanocomposites ${ }^{11,12}$. Therefore, we sought to investigate this potentially extremely important use of our nanohierarchical MOF films in pollution cleaning. The methodology and results are presented in the following.

Methodology: Some of the most hazardous pollutants with carcinogenic properties ${ }^{13,14}$, such as rhodamine $B$, benzene and toluene were selected to exploit and demonstrate the pollution cleaning capability of MOF film. Adsorption of rhodamine B was quantified using UV-vis spectroscopy (Shimadzu, UV2600i) by measuring the absorbance at $553 \mathrm{~nm}$. A stock solution of $1 \mathrm{mg} / \mathrm{mL}$ was prepared by dissolving rhodamine $B$ in water. The stock solution was, then, diluted $(0.62 \mu \mathrm{g} / \mathrm{mL}, 1.25 \mu \mathrm{g} / \mathrm{mL}, 2.5 \mu \mathrm{g} / \mathrm{mL}, 5 \mu \mathrm{g} / \mathrm{mL}$ and $10 \mu \mathrm{g} / \mathrm{mL})$ to acquire a calibration curve. For adsorption study, a MOF coated glass slide was dipped in a $50 \mathrm{~mL}$ solution of $10 \mu \mathrm{g} / \mathrm{mL}$ rhodamine $B$. The solution was subjected to absorbance measurement at predetermined intervals of $10 \mathrm{~min}, 30 \mathrm{~min}, 1 \mathrm{hr}, 2 \mathrm{hrs}$ and $4 \mathrm{hrs}$ by taking $2 \mathrm{~mL}$ each time from the solution at $25^{\circ} \mathrm{C}$.

The measurements of benzene and toluene were done in UCL Chemistry Mass Spectrometry Facility using a Thermo Scientific Trace 1300 Gas Chromatographer coupled to an ISQ mass spectrometer system set-up at selected ion monitoring mode. Thermo Scientific TR-5MS column $(30 \mathrm{~mm} \times 0.25 \mathrm{~mm}$ ) with $0.25 \mu \mathrm{m}$ film thickness was used for separation of benzene and toluene during the GC-MS run. Each solvent (analytical grade) was dissolved into water to prepare stock solution of $50 \mu \mathrm{g} / \mathrm{mL}$. The stock solution was further diluted to make calibration solutions of $10 \mathrm{ng} / \mathrm{mL}, 5 \mathrm{ng} / \mathrm{mL}, 2.5$ $\mathrm{ng} / \mathrm{mL}, 1.25 \mathrm{ng} / \mathrm{mL}$ and $0.62 \mathrm{ng} / \mathrm{mL}$ for both solvents. As above, coated substrates (75 $\mathrm{mm} \times 25 \mathrm{~mm}$ ) were dipped into $50 \mathrm{~mL}$ of $10 \mathrm{ng} / \mathrm{mL}$ solutions and the collected sample were injected into the GC-MS system at pre-determined time intervals of $10 \mathrm{~min}, 30 \mathrm{~min}$, $1 \mathrm{hr}, 2 \mathrm{hrs}$ and $4 \mathrm{hrs}$ at $25^{\circ} \mathrm{C}$.

Adsorption results: The adsorption of dye (rhodamine B) and organic compounds (benzene and toluene) was followed over time using UV-Vis and GC-MS spectroscopy and the results are presented in Fig. $3 \mathrm{H}$ and S20. Since the weight of adsorbent (MOFs) is unknown, the adsorption capacity is calculated simply as the difference of initial concentration and remaining concentration of the pollutant in the solution. The area of the substrate for adsorption experiments was $18.75 \mathrm{~cm}^{2}$. As shown in Fig. $3 \mathrm{H}$, the evolution of dye (rhodamine B) adsorption is slightly different from those of benzene and toluene. In case of rhodamine $B$, a significant increase in the adsorption was observed in the first 60 minutes followed by stabilisation whereas, the maximum adsorption of benzene and toluene occurred within the first 10 minutes. The adsorption of rhodamine can also be 
clearly observed with naked eye (see the photographs in Fig. S21A). However, for better understanding of the adsorption process, the results were fitted in pseudo-second-order kinetics:

$$
\frac{t}{q_{t}}=\frac{1}{k q_{e}^{2}}+\frac{t}{q_{e}} t
$$

where $q_{t}$ is the adsorption capacity at time $\mathrm{t}, q_{e}$ at equilibrium, and $\mathrm{k}$ is the rate constant of pseudo second order kinetics.

As shown in Fig. S21, the results fit the pseudo-second-order kinetic model very well $\left(\mathrm{R}^{2}\right.$ $>0.99$ ), which seems to indicate single-step surface adsorption with chemisorption being the rate limiting step.
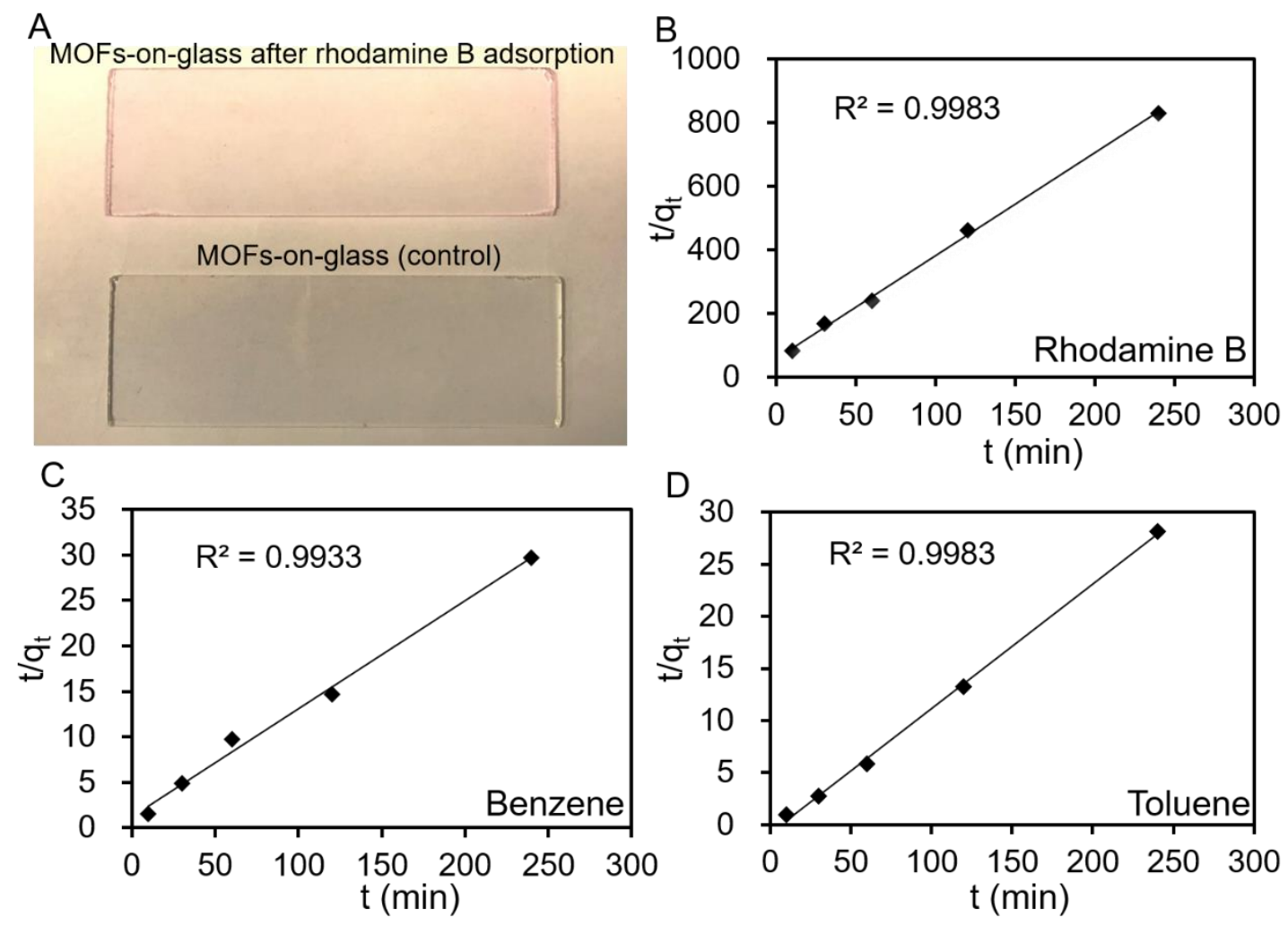

Figure S22. (A) Photographs showing visual change in the nanohierarchical MOF coated glass surface after adsorption of rhodamine $B$. The picture was taken after completion of adsorption experiments. (B) Rhodamine B, (C) benzene and (D) toluene absorbance fit to the pseudo-second order kinetics, i.e., eq. (2). 


\section{Caption information for videos 1-10}

Supplementary Movie 1: Water droplet sliding off the nanohierarchical MOF surface demonstrating excellent non-wetting behaviour.

Supplementary Movie 2: Free sliding of low surface tension liquid drops onto the nanohierarchical MOF surface. Specifically, butanone $(23.9 \mathrm{mN} / \mathrm{m})$, ethanol $(22.1$ $\mathrm{mN} / \mathrm{m})$, methanol $(22.5 \mathrm{mN} / \mathrm{m})$, acetone $(25.2 \mathrm{mN} / \mathrm{m}), 1$-butanol $(25 \mathrm{mN} / \mathrm{m}), 1$-decanol (28.5 $\mathrm{mN} / \mathrm{m})$, glycol $(47 . \mathrm{mN} / \mathrm{m})$, cyclohexanol $(33.4 \mathrm{mN} / \mathrm{m})$, 1,2-butanediol (37.2 $\mathrm{mN} / \mathrm{m})$ and sunflower oil $(\sim 33.4 \mathrm{mN} / \mathrm{m})$ were all seen to slide off at $15^{\circ}$ substrate angle.

Supplementary Movie 3: Self-cleaning of nanohierarchical MOF surface showing efficient removal of carbon dust with water droplets.

Supplementary Movie 4: Water droplets bouncing off the nanohierarchical MOF surface at two different velocities, $1.4 \mathrm{~m} / \mathrm{s}$ and $4.2 \mathrm{~m} / \mathrm{s}$, as labelled in the video. The spreading radius at higher impact velocity records 3 times larger than that falling at slow speed but contact time remained approximately similar.

Supplementary Movie 5: A water jet (nozzle $=0.5 \mathrm{~mm}$ ) impacting on the nanohierarchical MOF surface at $\sim 35 \mathrm{~m} / \mathrm{s}$ velocity to establish excellent resistance of the surface against liquid impalement. The jet impact test was repeated at least thrice at the same spot. The continuous sliding of the water droplets from the surface (e.g. droplets emanating the nozzle towards the end of the impact test, when the water in the syringe ran out) ruled out any pinning. However, to further evaluate the impalement resistance, drop mobility (as shown in supplementary movie 7 and 8) of water droplet was also tested.

Supplementary Movie 6: A water jet (nozzle $=2.5 \mathrm{~mm}$ ) impacting the nanohierarchical MOF surface at $\sim 35 \mathrm{~m} / \mathrm{s}$ velocity. The integrity of the surface after jet impact test was evaluated in the same way as explained above.

Supplementary Movie 7: A water jet (nozzle $=2.5 \mathrm{~mm}, \mathrm{~V}=\sim 35 \mathrm{~m} / \mathrm{s}$ ) test recorded by ordinary camera.

Supplementary Movie 8: Left: The water droplet sliding off from the spot of repeated jet impact tests. Right: The droplets remaining after jet impact test sliding off at $\leq 15^{\circ}$ angle, confirming excellent impalement resistance of the nanohierarchical MOF, up to jet speed of $\sim 35 \mathrm{~m} / \mathrm{s}$. 
Supplementary Movie 9: Water droplet impacting at $1.2 \mathrm{~m} / \mathrm{s}$ and bouncing off the nanohierarchical MOF surface even after 50 tape peel cycles, confirming the surface robustness.

Supplementary Movie 10: Free sliding of water droplets on nanohierarchical MOF surface after pencil hardness test.

\section{References}

1. M. Kandiah, M.H. Nilsen, Usseglio, S. Jakobsen, U. Olsbye, M. Tilset, C. Larabi, E.A. Quadrelli, F. Bonino, J.P. Lillerud, Synthesis and stability of tagged UiO-66 Zr-MOFs. Chem. Mater. 22, 6632-6640 (2010).

2. U. Betke, S. Proemmel, S. Rannabauer, A. Lieb, M. Scheffler, F. Scheffler, Silane functionalized open-celled ceramic foams as support structure in metal organic framework composite materials. Microporous Mesoporous Mater. 239, 209-220 (2017).

3. J. Liu, C. Wöll, Surface-supported metal-organic framework thin films: fabrication methods, applications, and challenges. Chem. Soc. Rev., 46, 5730-5770 (2017).

4. L. Wang, T.J. McCarthy. Covalently attached liquids: instant omniphobic surfaces with unprecedented repellency. Angew. Chem. Int. Ed. 55, 244-248 (2016).

5. C. Peng, Z. Chen, M.K. Tiwari. All-organic superhydrophobic coatings with mechanochemical robustness and liquid impalement resistance. Nature Materials, 17, 355-360 (2018).

6. L. Zhou, S. Wang, Y. Chen, C. Serre, Direct synthesis of robust hap UiO-66(Zr) MOF using poly(ethyleneterephthalate) waste as ligand source. Micropor. Mesopor. Mater. 290, 109674 (2019).

7. K. M. Zwolinski', M. J. Chmielewski, TEMPO-appended metal-organic frameworks as highly active, selective, and reusable catalysts for mild aerobic oxidation of alcohols. ACS Appl. Mater. Interfaces, 9, 33956-33967 (2017).

8. B.S. Yilbas, A. Al-Sharafi, H. Ali, N. Al-Aqeeli, Dynamics of a water droplet on a hydrophobic inclined surface: influence of droplet size and surface inclination angle on droplet rolling. RSC. Adv. 7, 48806-48818 (2017).

9. R.M. Amador, L. Cirre, M. Carboni, D. Meyer, BTEX removal from aqueous solution with hydrophobic $\mathrm{Zr}$ metal organic frameworks. J. Environ. Manage. 214, 17-22 (2018).

10. N.C. Burtch, H. Jasuja, K.S. Walton, Water stability and adsorption in metal-organic frameworks. Chem. Rev. 114, 10575-10612 (2014).

11.D.M. DeChellis, C.M. Negulle, D.T. Genna, Removal of hydrocarbon contaminants from water: exploiting hydrophobic UiO-66 derivatives. J. Mater. Chem. A, 8, 5848$5852(2020)$. 
12. C.P. Cabello, M.F. Picó, F. Maya, M. Rio, G.T. Palomino, UiO-66 derived etched carbon/polymer membranes: high-performance supports for the extraction of organic pollutants from water, Chem. Eng. J. 346, 85-93 (2018).

13. L.F. Lima, J. R. Andrade, M.G.C. da Silva, M.G.A. Vieira, Fixed bed adsorption of benzene, toluene, and xylene (BTX) contaminants from monocomponent and multicomponent solutions using a commercial organoclay, ACS Ind. Eng. Chem. Res. 56, 6326-6336 (2017).

14.K. Shen, M.A. Gondal, Removal of hazardous rhodamine dye from water by adsorption onto exhausted coffee ground. J. Saudi Chem. Soc. 21, S120-S127 (2017). 